\title{
Using high fidelity simulation to increase confidence in rehabilitation nurses
}

\author{
Sandra Stafford Cecil \\ Polytrauma Rehabilitation, VA Palo Alto, U.S.A. \\ Correspondence: Sandra Stafford Cecil. Address: 3801 Miranda Avenue, Palo Alto, California 94304, U.S.A. \\ Email: sandra.stafford-cecil@va.gov
}

Received: November 14, 2013

Accepted: August 26, $2014 \quad$ Online Published: September 14, 2014

DOI : $10.5430 /$ cns.v2n4p94

URL: http://dx.doi.org/10.5430/cns.v2n4p94

\section{Abstract}

Purpose: The purpose of this study was to explore nurses' reactions, competence and confidence levels before and after exposure to clinically relevant situations that could be addressed in simulation or in traditional learning environments.

Methods: A nonrandomized controlled study consisting of forty rehabilitation nurses was conducted at the VA Palo Alto Hospital in Palo Alto California. Nurses were placed in to either a control (C = Control) or intervention (I = Intervention) group. Each group of twenty would complete pre and post questionnaires using a Likert scale designed to test their level of confidence and comfortability after exposure to three scenarios found in the rehabilitation setting. The control group would receive information in a traditional format of power point lecture and interactive discussion, and the intervention group would be immersed in a reality-based environment using the simulation lab and a high fidelity mannequin. The intervention group would be filmed and debriefed after their experience. Data collected from the questionnaires would be collected and coded into an Excel spreadsheet to allow for export to a statistical software program to determine confidence interval and degree of change mean scores and effect size. A twelve month follow up was conducted to assess retention.

Results: Expected results differed from actual outcomes as there was little measurable difference between the intervention and control groups, although each group reported increased scores after both traditional and simulation learning. However, the twelve month follow up showed measurable increase in retention among the intervention group. Ordinal data was used with two independent groups. Non-parametric tests of significance were compared using a Wilcoxon test since normality could not be assumed. For comparison purposes, two sample $t$-tests were run, and the results were comparable to the Wilcoxon analysis. Power could not be determined because no true difference was known. Effect size of the possible benefit when introducing a new teaching method was calculated when appropriate.

Conclusions: The findings provide evidence that high fidelity simulation versus traditional problem based learning provides a greater sustained effect on confidence, competence and comfort levels among experienced rehabilitation nurses.

Clinical relevance: Competent nurses are associated with improved patient outcomes and competent care arises from knowledge applicable to practice. This study supports the importance of simulation training in increasing clinical competence and confidence.

\section{Key words}

Rehabilitation, Simulation, Nursing 


\section{Background}

Nursing comprises the largest segment of the healthcare interdisciplinary team. Competent nurses are associated with improved patient outcomes and competent care arises from knowledge applicable to practice. The question arises, how best to impart that knowledge? According to Parker ${ }^{[1]}$, "nurse educators are charged with the challenge of socializing students into the profession of nursing and providing them with opportunities to acquire the cognitive processes to practice”. Various teaching methodologies exist to prepare nursing students to practice, including simulation of clinical environments. But what of the experienced nurse? How does the nurse educator or clinical nurse leader empower the already practicing nurse to improve confidence and competence? According to the American Association of Colleges of Nursing landmark White Paper ${ }^{[2]}$, an effective educator uses appropriate teaching principles and strategies and current and emerging technologies in the care environment to support lifelong learning. If this is a keystone of future nursing education, which technologies are most effective in terms of retention and reflection?

The framework for this project arose out of a desire to use computer based simulation, as opposed to traditional problem based learning, to improve the education process for experienced rehabilitation nurses. Simulation as a continuing education method has been adopted as a best practice for several years in other professions. In his pioneering article on high fidelity computer simulation and anesthesiologists’ performance, Dr. David Gaba, director of the simulation program at the Palo Alto Veterans Administration hospital, quotes a dictionary definition of a simulator "as a training device that duplicates artificially the conditions likely to be encountered in some operation" ${ }^{[3]}$. He further states that "simulators have made their major impact as training devices in such diverse fields as commercial and military aviation, space flight, automotive driving, locomotive control, ship handling, fire-fighting, combat, and operation of nuclear power or petrochemical plants". If simulation is used successfully in other fields that are tasked with public safety, and in academic settings that train physicians and nurses, the question arose, could simulation education be incorporated in to the rehab practice setting to promote clinical competency and improve care processes?

The focus then became to develop a research study to test the hypothesis that computer based simulation learning (defined as videotaped simulated patient care situations using a high fidelity mannequin with subsequent debriefing) was superior to problem based learning (defined as lecture and discussion with information delivered via power point slides) because it allowed the practicing clinician to make mistakes in a safe environment and develop increased insight in to the complexities of nursing by honing critical thinking skills.The goal for this project was to create and implement a research trial that would test this hypothesis and produce valid and reliable data which could be repeated and replicated and ultimately be used as evidence to change practice. The first step was conducting a needs assessment. All the nurse managers and attending physicians in rehabilitation were queried via written survey, to see if they could identify clinically relevant situations that could be addressed in simulation. The issues identified were nurse to physician communication, recognizing an emergent situation, and defusing assaultive behavior. The goals then became to improve teamwork, standardize nurse to physician communication, and increase clinical reasoning. Specific teaching objectives were that participants would demonstrate increased confidence in ability to communicate with physicians, would be able to state the correct sequence of SBAR (situation, background, assessment, and recommendation) reporting, would be able to list the interventions necessary in an emergent situation, and would recognize verbal and non-verbal cues to defuse assaultive behavior. The addition of a Standardized Patient, or specially trained actor playing the part of an actual sick individual was included to enhance realism in the simulation setting and allow additional aspects of the real clinical world such as emotions and behavioral issues, to be explored. A study currently underway at the Palo Alto Veterans Administration involves the investigation of clinicians during realistic simulation and this pilot study was included under that existing IRB approval protocol. Consents to participate and videotape were completed, as well as confidentiality agreements to avoid contamination within the two groups. Funding was obtained from a Stanford Partnership for Spinal Cord Injury and Repair research grant. 
Table 1. Pre test (PRT) questionnaire

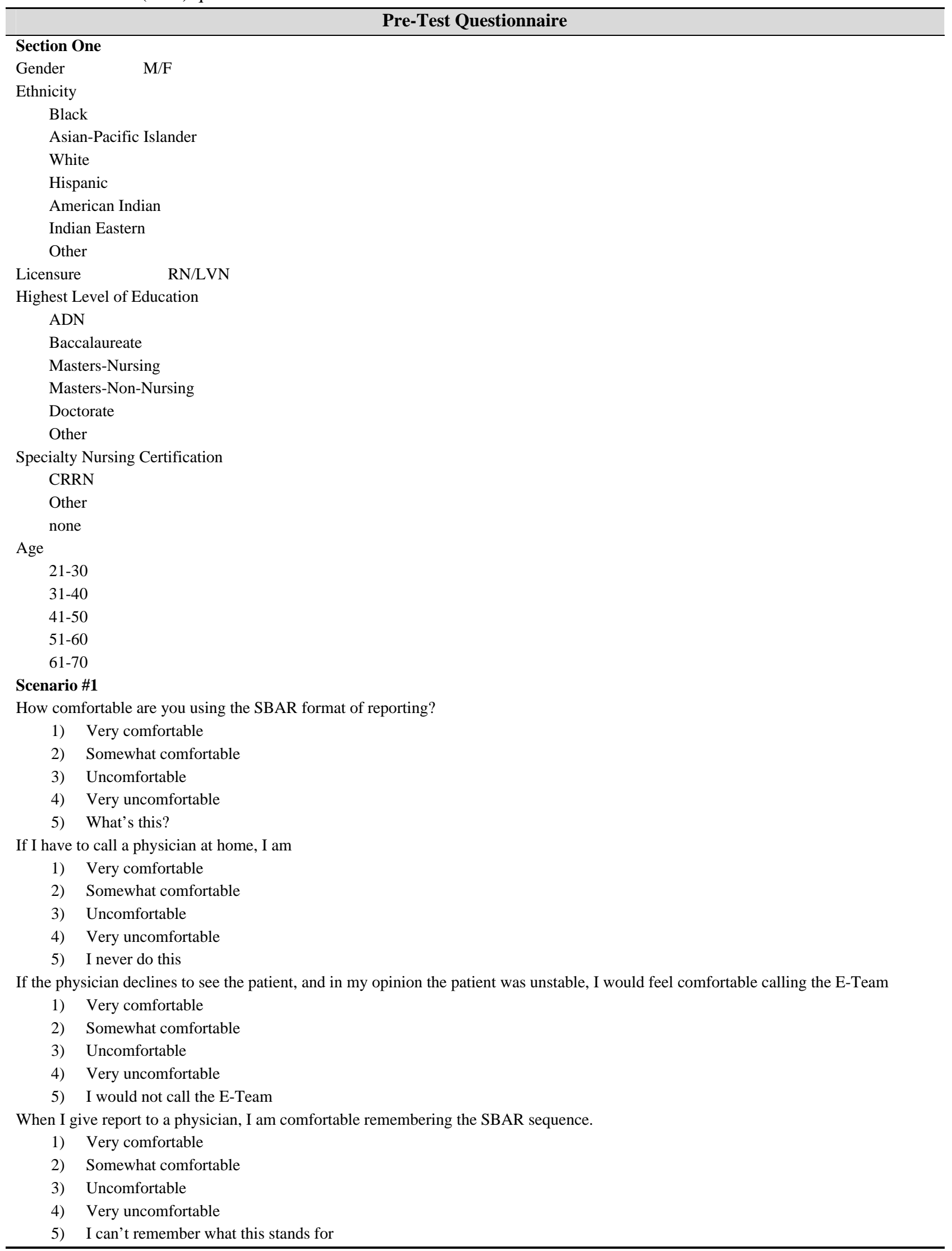


Table 1. (Continued)

\section{Pre-Test Questionnaire}

\section{Scenario \#2-}

How familiar are you with the contents of the crash cart and the operation of the defibrillator?

1) Very comfortable

2) Somewhat comfortable

3) Uncomfortable

4) Very uncomfortable

5) I have never used the crash cart or defibrillator

How comfortable are you that you would recognize the symptoms of Autonomic Dysreflexia in a patient?

1) Very comfortable

2) Somewhat comfortable

3) Uncomfortable

4) Very uncomfortable

5) I don't know what this is

I feel comfortable that I understand the role of each health care provider in a code situation.

1) Very comfortable

2) Somewhat comfortable

3) Uncomfortable

4) Very uncomfortable

5) I don't know what I should do in a code situation

How comfortable are you that you would recognize that a patient is becoming unstable medically?

1) Very comfortable

2) Somewhat comfortable

3) Uncomfortable

4) Very uncomfortable

5) I don't know what this is

\section{Scenario \#3-}

I feel comfortable recognizing verbal and non-verbal cues that a patient is becoming potentially assaultive

1) Very comfortable

2) Somewhat uncomfortable

3) Uncomfortable

4) Very uncomfortable

5) I don't know how to recognize this

I feel comfortable that I understand the concept of environmental modification

1) Very comfortable

2) Somewhat uncomfortable

3) Uncomfortable

4) Very uncomfortable

5) What's this?

I feel comfortable working in tandem with another care provider and an agitated, brain -injured patient

1) Very comfortable

2) Somewhat comfortable

3) Uncomfortable

4) Very uncomfortable

5) I would not do this

I feel confident that I have the skills to anticipate and respond to the needs of an agitated patient.

1) Very confident

2) Somewhat confident

3) Not very confident

4) I am not confident at all

5) I would not do this 


\subsection{Study design}

Various study design options were explored in consultation with the Chief Nurse of Research at the Palo Alto VA. It was decided a randomized controlled study consisting of forty rehabilitation nurses would be implemented to collect quantitative data. This approach is appropriate for testing a null hypothesis because it uses a comparative methodology to establish that there was some change after intervention. Nurses would be recruited from all four areas of rehab, including Spinal Cord Injury, Polytrauma, Western Blind Rehabilitation, and the Polytrauma Transitional Program. Nurses would be randomized in to either a control or intervention group. Each group of twenty would complete pre and post questionnaires using a Likert scale designed to test their level of confidence and comfortability after exposure to three scenarios found in the rehabilitation setting. The control group would receive information in a traditional format of power point lecture and interactive discussion, and the intervention group would be immersed in a reality - based environment using the simulation lab and a high fidelity mannequin. The intervention group would be filmed and debriefed after their experience. Data collected from the questionnaires would be collected and coded into an Excel spreadsheet to allow for export to a statistical software program to determine confidence interval and degree of change mean scores and effect size. Due to staffing barriers, true randomization was not possible and the control group ultimately consisted of seventeen participants, while the intervention group consisted of twenty three participants.

This research project took place in March 2011 and a 12 month follow up survey was conducted in April 2012. Forty rehabilitation nurses were placed into either control or intervention groups. Due to tour of duty scheduling conflicts, the control group size was reduced to seventeen participants, and the intervention group size increased to twenty three participants. The control groups received information via lecture and a power point presentation within their respective working environments, and the intervention group was tested in the simulation lab located on the Palo Alto VA campus. A literature review was conducted (key words: simulation, nursing, rehabilitation) prior to the study design to see if any data already existed to support using computer simulation to test competence or confidence in experienced nurses. Although a plethora of articles were found that test students in medical and nursing schools, very little has been published with regard to staff development in practicing nurses, including those in the specialty of rehabilitation. A second literature search on the role of computer based simulation and learning was then begun (key words: computer simulation, education, health care) and numerous examples were found listing the advantages of simulation in education. Innovative educational experiences are required to promote continued competence for experienced clinicians in an increasingly complex health care environment ${ }^{[4]}$. In an article written on the future vision of simulation in health care, Gaba writes, "Once a clinician has completed training, the required level of continuing education and training is often minimal and unstructured...part of the vision for the future is that clinical personnel, teams, and systems should undergo continual systematic training, rehearsal, performance assessment, and refinement in their practice” ${ }^{[5]}$. A third literature search on the relationship of time and learning retention (key words: long-term, retention of information, engaging lectures) was undertaken to see if time spent in a particular learning technique resulted in improved learning retention. In their comparison of traditional and engaging lecture methods ( defined as broken or interactive lectures with brainstorming sessions, open discussion, and real world situations). Miller, et al divided the same cohort of 120 first year dentistry students at the University of Louisville, School of Medicine, to allow a direct comparison of engaging physiology lectures versus didactic lecture formats in learning physiological systems. Time spent in curriculum was equal but the use of engaging lecture methods led to a statistically significant higher average on unit exams compared with didactic lectures ${ }^{[6]}$.

\subsection{Conceptual framework}

Transformative learning theory derives from the premise that today's adult learner needs to develop the ability to become an independent autonomous thinker ${ }^{[7]}$, which can be further conceptualized to three central themes: the role of experience, rational discourse, and critical reflection in knowledge development. Computer based simulation provides a foundation for transformative learning as participants develop their understanding of the situation through experiences. Mezirow further describes this process "as a frame of reference for a particular experience that is developed through an uncritical lens that allows individuals to create values, assumptions, and beliefs about a particular phenomenon: a meaning scheme that 
includes constellations of concepts ,beliefs, judgments, and feelings which shape a particular interpretation” ${ }^{[7]}$. Thus, transformative learners use their new perspective when they subsequently engage in new situations and develop new ways of interpreting disorienting dilemmas. Simulation learning has the potential to promote transformative learning in clinical situations through its use of reflection, post scenario debriefing, video feedback, and interaction within a group setting of peers.

\section{Methodology}

Forty registered or licensed vocational nurses currently practicing in rehabilitation environments were voluntarily recruited to participate during the month of March 2011. The original study design called for nurses to be randomly allocated to either a high fidelity simulation ( $\mathrm{I}=$ Intervention) group or lecture group ( $\mathrm{C}=$ Control), but tour of duty and staffing constraints made true randomization impossible and nurses were assigned based on availability to participate. All nurses completed research consents, confidentiality agreements, and pre test questionnaires. The simulation/intervention group received an orientation to the simulation center and mannequin features and a "ghost" was present in the room during scenarios to assist with supply procurement. The simulation mannequin is a high-fidelity, life sized human patient simulator with features such as built-in physiologic models, realistic eye movements, spontaneous breathing and voice capability. The simulator is controlled from an adjacent control room. Video cameras allow viewing and videotaping of scenarios from different angles and participants wore microphones to facilitate audio recordings. Communication between the simulated patient room and the control room is made possible through an overhead speaker and/or a telephone. Participants in the control group received a power point lecture focusing on an overview of the scope of practice of rehabilitation nursing and then an introduction to each scenario. Group discussion and interaction were encouraged as each participant verbalized appropriate clinical interventions a nurse would be expected to perform and the critical thinking processes guiding the expected behaviors. Subjects in both groups were asked to complete a post test confidence questionnaire using a five point Likert scale, self-assessing their knowledge about, and confidence in, three situations found in rehabilitation nursing. The tool was developed using both questions and statements that participants would rate agreement with. Competence would be measured by a list of expected clinical actions participants would describe or perform. The pre-test questionnaire is displayed in Table 1 . This same questionnaire was distributed twelve months later to determine retention.

\section{Definitions}

- Comfortability - "free from vexation or doubt”, Random House College dictionary ${ }^{[8]}$.

- Competence - "The state or quality of being adequately or well qualified" ${ }^{[8]}$.

- Confidence - "faith or belief that one will act in a right, proper, or effective way" ${ }^{[8]}$.

- Control group (C)-17 experienced rehabilitation nurses completing pre and post exposure questionnaires measuring confidence and comfort levels after listening to three scenarios delivered in a power point presentation.

- Intervention group (I)-23 experienced rehabilitation nurses completing pre and post exposure questionnaires measuring confidence and comfort levels after participating in same three scenarios using mannequin based simulation with video feedback and de-briefing.

- $\quad$ Pre-test (PRT) - Demographic information and 12 point ( identified as \#1-\#12) questionnaire using Likert 1-5 scale self rating confidence.

- $\quad$ Post-test (POT) same questionnaire as above minus the demographic information.

The simulation/intervention group participants acted individually in the first two scenarios and then were allowed to call for help in scenario \#3 to gauge ability to work in tandem with another care giver. The second responder was sequestered 
and arrived mid-scenario. Also, the third scenario involved the use of a Standardized Patient, or trained actor to promote realistic interaction with a brain injured and potentially assaultive patient. Other members of each intervention group were watching the scenario via video in the debriefing room. Subjects in the lecture/control group were given the same scenarios as the simulation/intervention group. The total time for completing the scenarios were significantly shorter in the control group (65 minutes) then the intervention group (3 hours). De-briefing, or post-scenario discussion times were also shorter in the control group, although the number of participants in each group was relatively consistent (4 or 5 ) and there were no time limitations imposed. The intervention groups participated and observed the other groups.

Each scenario had the same teaching objectives for both intervention and control groups. After the intervention scenarios, experienced debriefers provided scenario specific, and simulation learning in general, feedback to each participant. Participants in both groups then completed a post test questionnaire (POT) identical to the pre-test minus the demographic information.

\section{Statistics}

Results from the pre and post questionnaire were entered in to a database using Excel formatting. Each question corresponded to a 1-5 number answer. However, the \#5 response became evident as more of a "N/A" rather than an added level of discomfort as LVNs would not have this action in their routine scope of practice i.e. calling a physician at home. The Likert scale \#5 responses were then eliminated and the calculations were re-run with a maximum score of 4's for a more true measure of comfort without the skew of ignorance. Thus, the minimum score possible for each question was four ( all \#1's) and the maximum was sixteen (all \#4's). This database was then exported to JMP 8 by SAS Institute, Cary, North Carolina for statistical analysis. A $p<0.005$ was considered statistically significant. The final $n=40$ subjects, 23 in the intervention (I) group and 17 in the lecture (C) group. Ordinal data was used with two independent groups. Nonparametric tests of significance were compared using a Wilcoxon test since normality could not be assumed. For comparison purposes, two sample t-tests were run, and the results were comparable to the Wilcoxon analysis (see NPRT1 and POT 1). Power could not be determined because no true difference was known. The effect size of the possible benefit when introducing a new teaching method was calculated when appropriate.

\section{Results}

Demographic data collected showed an age range from 21-70 years with a mean participant age of $39+2.4$ years and a gender ratio of 7 males and 33 females. There were 10 licensed vocational nurses (LVNs) and 30 Registered nurses (RNs) with 16 holding BSN degrees, 12 holding AD degrees 1 MSN, and 1 FNP. 6 nurses held specialty certifications as Certified Rehabilitation Registered Nurses (CRRN). All four areas of rehab were represented with participants from each in both the control and intervention groups. The predominant ethnicity (19) was Asian/Pacific Islander. In the selfassessment pre-test of confidence, the control group rated themselves as more comfortable in all scenarios and thus, could achieve less, moving towards 1 or very comfortable. The exception was pre test question \#1 (PRT1) on degree of comfort with SBAR reporting as illustrated below. The total time for completing the scenarios were significantly shorter in the control group (65 minutes) then the intervention group (3 hours). Expected results differed from actual outcomes as there was little measurable difference between the intervention and control groups, although both groups reported increased scores after both traditional and simulation learning. However, the twelve month follow up survey, which was conducted via identical post test questionnaire submitted to original forty nurses and returned by twenty five, showed best retention of information among the intervention group. Four questions were asked for each of the three clinically relevant scenarios (using SBAR format, recognizing an emergent situation, and defusing assaultive behavior in an aggressive patient) in the pre-test, the post-test, and the 12 month follow up and the scores were then summed and the mean response was calculated. Lower scores indicated high competence/confidence and higher scores indicated decreased competence/ confidence in ability. 
In the first question (PRT1/POT1) regarding comfortability with the SBAR report format, both groups gained increased confidence and competence, although the control group reported higher gains. In the intervention group, 13 out of 23 participants reported 2's, or somewhat comfortable, and moved to 121 's or very comfortable after scenario exposure. $50 \%$ of the control group (8 participants) started as 4's, then 7 participants moved to 2 or 1 (somewhat comfortable or very comfortable) on the post test. Similarly, in Question 2 (PRT2/POT) that dealt with comfort level when calling a physician at home, both groups reported gains after scenario exposure, but the increase was less pronounced in the intervention group. For Question 6 (PRT6) that dealt with recognizing the symptoms of Autonomic Dysreflexia, a potentially life threatening condition, there was a significant increase in both groups. Again, the control group starting out with higher pre-test confidence levels and yet still showed improvement after scenario exposure. In the intervention group, a 34\% improvement in confidence levels was reported in post testing (POT6). Questions 9 (PRT9) and 12 (PRT12) dealt with recognizing the verbal and non-verbal cues in a brain injured patient that is becoming potentially assaultive. This scenario used a professional actor or, Standardized Patient, to enhance the realism during the simulation intervention. Here the pre-test results were almost identical for both the control and intervention groups with a slight difference in gender, as males rated slightly lower than females. (48\% F to $43 \% \mathrm{M}$ on 1's or very comfortable). This study had 7 males and 33 females. The improvement was also almost identical for both groups as evidenced by the post-test scores (pot9). Thus, no matter how the content was delivered, exposure to information about recognition of cues that a patient is becoming potentially assaultive increases confidence and competence as self-reported. The final question (PRT12) again dealt with brain injured patients and aggressive behaviors and having the skills to anticipate and respond appropriately. Both groups were mostly $\{2$ 's $\}$ or somewhat confident with the control group reporting $53 \%\{2$ 's $\}, 18 \%\{3$ 's $\}$ not very confident, and $29 \%$ very confident. The intervention group reported $87 \%\{2$ 's $\}$, with $9 \%\{1$ 's $\}$, zero $\{3$ 's $\}$, and $4 \%\{4$ 's $\}$ or I am not confident at all. In the post test analysis, the control group jumped to $71 \%\{1$ 's $\}, 29 \%\{2$ 's $\}$, and zero \{3's $\}$, while the intervention group reported $52 \%\{1$ 's $\}, 43 \%\{2$ 's $\}$, and $4 \%\{3$ 's $\}$.

\section{Twelve month follow up}

The twelve month follow up questionnaire showed significant retention among the intervention group as evidenced by this bar graph (see Figure 1).

Figure 1. Mean response after 1 Yr. follow up

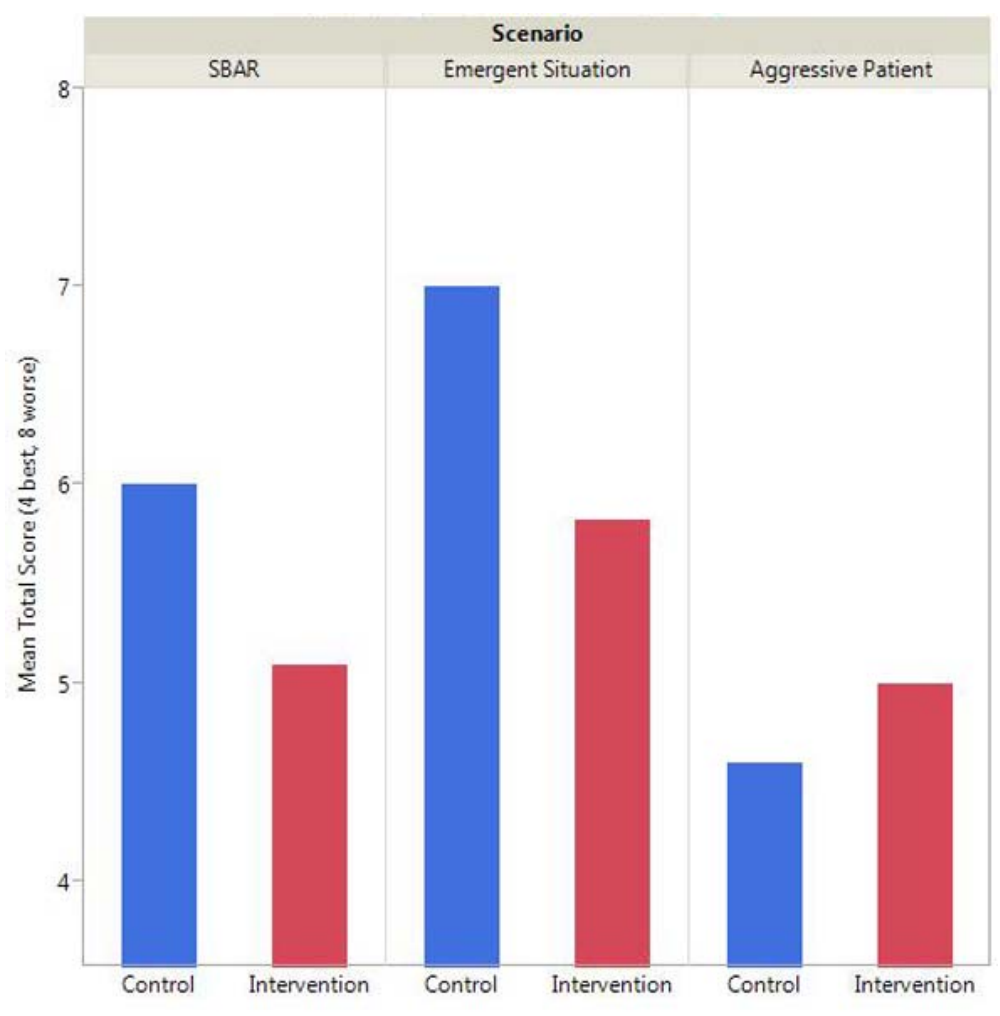

Published by Sciedu Press 
In reviewing the 12 month follow up, in both the SBAR and Emergent situation scenarios there was a clear difference in scores, but due to small sample size, I was unable to show statistical (i.e. $p<0.1$ ) significance. However in the Aggressive Patient scenario there was no 12 month difference. Does this speak to the reality of confidence levels after dealing with a live, agitated, actor versus a theoretical situation presented in a power point lecture? Had the nurses recently dealt with an aggressive patient in a real clinical situation and thus had higher confidence and competence in their abiity to deal with this type of patient? More research is needed to explore these differences in confidence.

\section{Discussion}

Even if formal evidence was limited, comments made by participants in the study were overwhelmingly supportive of simulation as an effective and transformative learning experience. One respondent in the intervention group remarked, “this was an initially a more stressful way to learn, but I definitely won't forget it. It was even a little bit fun, once I relaxed." These comments were echoed by both male and female participants, and with nurses in all age ranges, although nurses with less years of experience seemed more comfortable with the simulation experience and commented that "it was like being back in nursing school, only with a much better mannequin" and "simulation in school was kind of dry, at least here it was hard, but I really learned something and it was fun". Several participants commented on the realism of the mannequin and the ease with which they suspended disbelief, but one participant was unnerved by the mannequin's features and stated "It was just too weird, with him blinking and talking and everything. I just froze and I couldn't think. I just didn't know what I was expected to do". Nurse educators planning to incorporate simulation learning in to their curriculum need to be aware that some participants will be extremely reluctant to the point of refusal, and may need repeated exposure to simulation to decrease discomfort and self-criticism, or alternate immersion experiences. The inability to completely replicate a human patient was eliminated in the third scenario, in which the participants expect to walk in to the room and see a mannequin, but are instead confronted with a live human being, a SP or, Standardized Patient. The actor is briefed on the scenario and told to act a certain way in response to the nurse's interventions. For example, as his agitation increases, he begins to pace and shout, and at one point grabs a pair of scissors which are laying on a bedside table. The nurse needs to balance the issue of staff safety with patient safety and participants commented on how truly realistic this issue is. "It was like being back on the ward, my heart was really racing". "This guy was really good, he totally reminded me of the brain injured soldiers we get”.

A recurring theme that was expressed during the debriefing sessions was the nurse's fear of making an error and "getting in trouble”. One nurse stated, “I wasn't sure if the doctor would yell at me or not if I called him, but I decided to anyway because I thought he ought to know about the situation”. This lack of confidence was more pronounced in the younger nurses (21-30) and underscores the importance of establishing a collegial and trusting culture within health care for effective and timely communication. Another emerging theme was the nurses' lack of innovation in dealing with a dynamic situation where all the causative features are unknown. This is discussed in the debriefing sessions as "thinking about Plan B, or even C "This strict reliance on protocol and failure to reevaluate when initial interpretations are wrong is exactly the patient safety issue that simulation is best suited for, where mistakes can be made in a safe environment and reflective discourse allows for truly critical thinking and transformative learning. Being able to observe herself with actual patient care in progress caused one nurse to comment, "I'm better than that in real life". This underlies another strength of simulation, the use of audio and visual recordings which can be a "window in to your practice" as described by Dr. Steven Howard, co-director of the simulation program at the Palo Alto VA. He further stated, "It's the little things that save lives. You're the one that knows the patient. How do you access the inert intelligence that you possess as an experienced provider in a time of stress?' Again, the strength of simulation is the ability to rehearse and review common, uncommon, and unusual situations to enhance preparation and competence. Transformative learning occurs when the learner incorporates conceptual explanations and past experiences in to every day practice and changes behavior. Simulation allows for a creative integration of old and new behaviors and the verbal and visual feedback invites reflection. As these reflections and new behaviors become standard practice, confidence in the ability to handle and respond effectively improves. If competence is equal parts skill , knowledge, and execution, then allowing the learner to practice physical and 
intellectual experiences in a structured environment such as simulation will have a lasting impact in increasing competence.

\section{Summary}

This nonrandomized controlled study looked at computer based simulation versus traditional problem based learning and its effect on confidence and competence levels among experienced rehabilitation nurses. Expected results were not borne out by the statistical metrics as there were no significant differences between starting and end mean scores between the control and intervention groups. However, the twelve month follow up questionnaire showed significant retention among the intervention group versus the control group in terms of continued confidence in ability to react in certain scenarios. This finding may have been a result of the difference in exposure time between the control and intervention groups (65 minutes versus 3 hours). Or perhaps the variable of time is less important than engagement of the learner. Given the constraints of instructor preparation and protected educational time and money available for nursing staff, restructuring curriculum to incorporate blended learning methods that combine multiple modalities may give the "most bang for the buck" and improve both the qualitive experiences and the retention of learning.

This study had several limitations: True random allocation did not occur as both groups were not equivalent except for the operation of chance, so any differences in the outcome cannot be directly attributed to the intervention. A small sample size $(n=40)$ makes generalization problematic and there were difficulties with an even smaller sample size (15 respondents were lost to follow up thus only 25 of original 40 nurses responded) in the 12 month follow up questionnaire. The data was obtained by self-assessment which is inherently biased and subjective. Some nurses who were exemplary in their performance still rated themselves as 3 and 4 in pre tests, while others who had clearly struggled, rated themselves as 1 and 2. Further studies are needed to determine the best way to assess individual levels of confidence and competence, and the relationship between comfortability, confidence, and competence. Methodological rigor was weakened as power could not be determined because no true difference was known. A small sample size made certain Chi Square tests suspect. Finally, most of the participants in the intervention group were day shift nurses, although 5 of the 23 also work evening shifts. All of the nurses in the control group were evening or night shift workers. Does this difference in primary tour of duty affect confidence levels and nurse-physician communication? Does less interaction with other members affect clinical knowledge through fewer teaching opportunities (i.e. nurse/physician rounds) and less cross-pollination? Does this affect competence? Additional studies in simulation's role in staff development and teamwork are needed and the researcher needs to be acutely aware of the 24 hour nature of nurses' work, remaining sensitive to patient care staffing demands that may preclude participation on a particular shift. There was also an unavoidable difference in time spent within each group. Both groups reported increased confidence after either form of instruction, leading to the conclusion that all forms of instruction are valuable. For example, in the SBAR scenario there was global improvement in both groups on the post test but less retention 12 months later. If transformative learning is measured by sustained changes in practice over time, a restructuring of traditional educational methods may result in even greater improvements. Finally, confidence correlates to competence. Competency is a combination of knowledge and skills that enable a provider to make the appropriate intervention. Simulation provides a hand-on learning environment in which to practice and refine skills and behaviors. Valuable insight can arise from recognition of clinicians' strengths and weaknesses. This reflection may lead to retention and transformative learning. Although the results of this study were surprising in the lack of statistical difference between control and intervention groups, scenario based simulation is an innovative and potentially powerful way for experienced nurses to improve performance and enhance confidence and competence. In an editorial written over twenty years ago, Dr. Gaba states "no industry in which human lives depend on the skilled performance of responsible operators has waited for unequivocal proof of the benefits of simulation before embracing it" ${ }^{[3]}$.

\section{References}

[1] Parker B. Transformative learning as a context for human patient simulation. Journal of Nursing Education. 2009 ; 49: $326-332$.

Published by Sciedu Press 
PMid:20210271 http://dx.doi.org/10.3928/01484834-20100224-02

[2] American Association of Colleges of Nursing ( AACN) White paper on the education and role of the clinical nurse leader. 2007 Feb; 13: 84.

[3] Gaba David M. Improving anesthesiologists’ performance by simulating reality. Anesthesiology. 1992; 76: $491-494$. http://dx.doi.org/10.1097/00000542-199204000-00001

[4] Waxman KT. The development of evidence-based clinical simulation scenarios: Guidelines for nurse educators. Journal of Nursing Education; 2010; 49: 29-35. PMid:19810672 http://dx.doi.org/10.3928/01484834-20090916-07

[5] Gaba David M. The future vision of simulation in healthcare. Quality Safety in Health Care. 2004; 13 (Supp 1): 2-10. http://dx.doi.org/10.1136/qshc.2004.009878

[6] Miller CJ. A comparison of traditional and engaging lecture methods in a large,professional-level course.Advanced Physiological Education. 2013 Dec; 37(4): 347-355. PMid:24292912 http://dx.doi.org/10.1152/advan.00050.2013

[7] Mezirow J. Transformative learning: Theory to practice. P.Cranton ed.Transformative learning in action: Insights from practice. New York: John Wileyand Sons; 1995: 5-12.

[8] Random House College dictionary. Revised ed. New York: Random House; 2008; 268-282p.

[9] Norman G, Schmidt HG. Effectiveness of problem-based learning curricula: theory,practice, and paper darts. Medical Education 2000; 34: 721-728. http://dx.doi.org/10.1046/j.1365-2923.2000.00749.x

[10] Oja KJ. Using Problem-Based Learning in the Clinical Setting to Improve Nursing Students' Critical Thinking: An Evidence Review; Journal of Nursing Education. 2011; 50: 145-151. PMid:21210603 http://dx.doi.org/10.3928/01484834-20101230-10

[11] Wetzel SK, Konoske PI, Montaque WE. Estimating skill degradation for aviation antisubmarine warfare operators (AWs): Loss of skill and knowledge following training (Tech Rep No NPRDC TR-83-31). San Diego: Navy Personnel Research and Development Center; 1983: 1-21. 